\title{
Neurologic Wilson's disease
}

\author{
Matthew T. Lorincz \\ Department of Neurology, University of Michigan Health Systems, Ann Arbor, Michigan, USA \\ Address for correspondence: Matthew T. Lorincz, M.D., Ph.D., Assistant Professor, University of Michigan Health Systems, \\ Department of Neurology, 1500 E. Medical Center Drive, SPC 5316, Ann Arbor, MI 48109-5316. Voice: 734-615-8234; \\ fax: 734-615-4991. Lorincz@umich.edu
}

\begin{abstract}
Despite a long history, Wilson's disease, an autosomal recessive disease caused by mutations in the ATP7B gene, remains a commonly misdiagnosed import disease. Mutations in ATP7B result in abnormal copper metabolism and subsequent toxic accumulation of copper. Clinical manifestations of neurologic Wilson's disease include variable combinations of dysarthria, dystonia, tremor, and choreoathetosis. Among neurodegenerative diseases, it is unusual in that misdiagnosis and delay in treatment are clinically relevant because treatments can prevent and cure Wilson's disease, if they are given appropriately. If left untreated, Wilson's disease progresses to hepatic failure or severe neurologic disability and death, while those adequately treated have normal life spans. This review focuses on the neurologic features of Wilson's disease, its diagnosis, and treatment options.
\end{abstract}

Keywords: ceruloplasmin; copper; dysarthria; dystonia; tremor; Parkinsonism; movement disorders

\section{The history of neurologic Wilson's disease}

The landmark paper in this disease was written in 1912 by Samuel Alexander Kinnier-Wilson, an American neurologist working at The National Hospital at Queen Square in London. ${ }^{1}$ He described a neurologic disorder associated with progressive lenticular degeneration of the brain and cirrhosis of the liver that came later to be known as Wilson's disease, or hepatolenticular degeneration. Prior to Wilson's description, Kayser in 1902 had described pigmented corneal rings, later to bear his name, in a patient believed affected by Wilson's disease. Wilson did not initially believe the connection of Kayser-Fleischer rings or liver pathology to the disease that bears his name. The disease described by Wilson was one dominated by juvenile age at onset with dystonia and contrasts to the form of disease described by Westpahl in 1883 as pseudosclerotic, having a young-adult age of onset and symptomatically consisting principally of tremor and dysarthria. Only later was parkinsonism recognized as a major clinical feature of Wilson's disease.

A connection to copper metabolism may have first been described in 1913 and was strengthened by observations of sunflower cataracts in Wilson's disease. This type of cataract had been recognized as being produced by copper-containing foreign bodies. Subsequently, in 1929 and 1930 excess brain and liver copper was described. ${ }^{2}$ In 1952 ceruloplasmin was demonstrated to be low in Wilson's patients. ${ }^{3}$ From 1963 through the mid 1970s use of radiocopper tracing by Walshe and colleagues demonstrated that in presymptomatic disease the liver is able to sequester injected copper. In symptomatic patients, injected copper was unable to be bound in the liver and was found in other tissues including the brain. ${ }^{4,5}$ A low biliary excretion of copper was later shown to be responsible for a failure to regulate copper balance. An autosomal inheritance pattern was delineated by Hall in 1921 and was confirmed by Bearn in $1960 .^{6}$ The molecular era of medicine brought the chromosomal localization of the Wilson's disease gene to chromosome 13q14.3 and identification of the causative gene, ATP7B a copper transporting P-type transmembrane ATPase. ${ }^{7-9}$

Cumings, who was the first to postulate an etiologic role for copper, suggested that chelation therapy with British antilewisite (BAL or dimercaprol) might be of benefit. ${ }^{10}$ The agent had been used during World War II as an agent to treat lewisite, an arsenic poison. By 1951, Cumings, Denny-Brown, 
and Porter described benefits of BAL in treatment of Wilson's disease. ${ }^{11,12}$ Alleviation of the painful BAL injections and their associated toxicity became possible with the description by Walshe in 1956 of the potential use of penicillamine to chelate copper in Wilson's disease. ${ }^{13}$ Early in the 1960s use of zinc as a decoppering agent was first undertaken by Schouwink and Hoogenrand, findings that have subsequently been borne out by larger trials. ${ }^{14,15}$ Seeking an alternate to penicillamine therapy, in 1969 Walshe began using trientine and has subsequently reported its beneficial effects in those refractory to penicillamine. ${ }^{16}$ As had been observed with zinc, molybdenum was know to induce copper deficiency in sheep. This observation eventually lead Walshe, in 1986, to consider its use in Wilson's disease. The effectiveness of tetrathiomolybdate as a treatment of Wilson's disease has now been confirmed. ${ }^{17-20}$

\section{Epidemiology}

Wilson's disease appears to be typical of rare autosomal recessive diseases in that it is present at a low frequency in all populations. An estimate for the disease frequency in most populations is about 17 per million, which would lead to a carrier frequency of 1 in $122 .{ }^{21}$ As with most autosomal recessive diseases, there may be pockets of excess Wilson's disease produced by founder effects, particularly if consanguity is common in the population. ${ }^{22}$

\section{Clinical manifestations}

During early life, the patient is presymptomatic but is accumulating copper, which invariably causes subclinical liver disease. Then, between early childhood and the fifth or sixth decade of life, but with a peak incidence of around 17 years, the patient presents with hepatic, neurologic, and psychiatric manifestations. ${ }^{23}$ It has been suggested that the hepatic, neurologic, and psychiatric presentations of Wilson's disease occur in roughly equal proportions. An accurate estimate of the presenting proportions is challenging because many of the large case series have ascertainment bias based on clinical specialty, and likely underrepresent the psychiatric presentation. Those with a primarily hepatic presentation appear to have an earlier age at presentation than those with the primarily neurologic presentation. ${ }^{23,24}$ Although Wilson's disease usually presents in childhood into early adulthood, a wider range of age at onset is recognized. Generally, is extremely rare for Wilson's disease to present after age 35, but late-onset cases continued to be reported. ${ }^{25}$ This review deals primarily with neurologic Wilson's disease, and hepatic manifestations will be briefly considered. Excellent descriptions of hepatic Wilson's disease can be found elsewhere. ${ }^{22,23,26}$

\section{Neurologic manifestations}

\section{Neurologic subtypes}

Walshe, who introduced penicillamine, trientine, and tetrathiomolybdate therapy, stated that "no two patients are ever the same, even in a sibship," and that there is no such thing as a typical picture of Wilson's disease. ${ }^{27}$ Mean age at onset of neurologic symptoms from large case series range from about $15-21$ years of age. ${ }^{24,273} \mathrm{Neu}-$ rologic manifestations at initial presentation have been reported in approximately $18-68 \% .^{27,29-31,34}$ The main clinical categories of neurologic Wilson's disease have been variably divided by neurologic presentation and signs. The clinical categories that encompass the majority of neurologic Wilson's disease are, dysarthric, dystonic, tremor, pseudosclerotic (tremor $+/$ - dysartrria), or parkinsonian. ${ }^{24,27-33,35-37}$ It is not uncommon for a single manifestation such as tremor, dysarthria, dystonia, or less frequently parkinsonism to be present at the initiation of the symptomatic period. As the disease progresses, it is typical for complex combinations of neurologic symptoms and signs to coexist in a single patient, with a small subset of features predominating. During the course of the disease, other neurologic features include, chorea, athetosis, myoclonus, seizures, ataxia, pyramidal signs, drooling, and eye movement abnormalities. ${ }^{27}$

\section{Neurologic features}

\section{Dysarthria}

Dysarthria is probably the most common neurologic manifestation of Wilson's disease. In large series that delineate dysarthria as a feature of Wilson's disease, it has been found in $85-97 \%$ of those with neurologic Wilson's disease..$^{29,31,37}$ Dysarthria in Wilson's disease is most frequently of the mixed type with varying spastic, ataxic, hypokinetic, and dystonic components. ${ }^{30,31,38}$ Speech involvement is 
frequently concordant with the neurologic involvement in individual patients. In those with dystonia, speech frequently will have dystonic qualities with a strained or harsh quality. In those with parkinsonism, the speech quality may have hypokinetic properties. ${ }^{39}$ Ataxic dysarthria, with variation in word spacing and volume, is often found in association with other types of dysarthria and may be more common in those with tremor. ${ }^{31}$

\section{Dystonia}

Dystonia can be focal, segmental, multifocal, or generalized and ranges in severity from mild to debilitating. ${ }^{38}$ Dystonia is a common finding in Wilson's disease, reported to be present in about 11$65 \% .{ }^{29-31,33,34,38}$ A common focal dystonic manifestation of Wilson's disease is the dystonic facial expression known as risus sardonicus. This type of dystonia inflicts the patient with a forced, often exaggerated, smile. ${ }^{38}$ Focal dystonia of the vocal cords, muscle of articulation, and swallowing frequently results in dysphonia, dysarthria, and dysphagia. Focal dystonia of the vocal cords, muscle of articulation, and swallowing can be an initial isolated feature or part of a generalized dystonia. Other focal dystonias include blepharospasm, cervical dystonia (torticollis), and writer's cramp. As the disease progresses focal dystonia can progress to segmental, multifocal, hemidystonia, and generalized dystonia. Dystonia, like many neurologic features of Wilson's disease, typically has a unilateral onset or predominance, but can progress to bilateral or generalized involvement. Severe dystonia may lead to extreme posturing of the trunk, neck, or extremities. As in other forms of dystonia, sensory tricks can often lead to temporary improvement. The presence of dystonia has been demonstrated to correlate with MRI signal abnormalities in the putamen. ${ }^{31,38}$

\section{Tremor}

Wilsonian tremor has been reported to be present in $22-55 \%$ and can occur at rest, upon assumption of a posture, or with action. ${ }^{27,29,30,32,34} \mathrm{Al}$ though widely known, the wing-beating tremor of Wilson's disease does not appear to be the most frequent tremor type. Early in the neurologic presentation of Wilson's disease, the tremor can be identical to the tremor of essential tremor, with the arms most frequently involved but also involving the head and legs. Persistence of tremor asym- metry and absence of voice tremor may differentiate this type of Wilsonian tremor from essential tremor, which is typically symmetric and frequently involves the voice. As the disease progresses, Wilsonian tremor may take on characteristics atypical of essential tremor. It is not uncommon to encounter a tremor with multiple position- and task-dependent characteristics in an individual patient. ${ }^{32}$ The kinetic tremor is most frequently a distal upper extremity, low amplitude, medium-to-high frequency tremor. The classical posture-induced wing-beating tremor, thought to be associated with lesions of the dentadorubrothalamic pathway, is a less frequently observed, lower frequency higher amplitude proximal upper extremity tremor elicited by holding the arms extended laterally or with the arms held in front with flexed elbows and palms facing downward. The wing-beating tremor is typified by increasing amplitude with increased duration of posture holding. ${ }^{2}$ A unilateral isolated rest tremor is atypical in Wilson's disease. When rest tremor is present it is usually accompanied by postural and kinetic tremor, which may be more severe than the rest tremor. ${ }^{29}$

\section{Parkinsonism}

In series in which parkinsonism has been considered as a separate symptom category, it has been reported in 19-62\%. ${ }^{27,31,33,34}$ Bradykinesia, imbalance, and cogwheel rigidity are the more common parkinsonian features. Unilateral rest tremor and parkinsonism are rarely isolated clinical feature of Wilson's disease, but like idiopathic Parkinson's disease, rigidity, and tremor are typically asymmetric.

\section{Choreoathetosis}

Chorea and athetosis have been reported to occur in $6-16 \%$ of those with neurologic Wilson's disease. ${ }^{29-31,33,34,37}$ Chorea is characterized by involuntary irregular rapid movements of the face, head, trunk, and extremities that are superimposed on and interrupt normal movement. Choreic movements can be subtle and small, occurring distally in the fingers resembling piano playing movements, and may also resemble fidgetiness and be incorporated into apparently purposeful movements. In an extreme form, they can manifest as disabling uncontrolled flailing movements of the extremities, termed ballism. Chorea is frequently accompanied by athetosis, a slow writhing movement of the limbs, trunk, or neck, and this combination is referred to as 
choreoathetosis. When present in Wilson's disease, chorea is more common in young onset disease (16 years of age and younger), where it has been reported in $20 \%$. In contrast, chorea was reported in only $3 \%$ of adult onset ( 17 years of age and older). ${ }^{27}$ Chorea can have many causes ${ }^{40}$ and is not usually present in isolation in neurologic Wilson's disease.

\section{Ataxia}

Ataxia has been reported as a common feature in Wilson's disease, present in around $30 \%,{ }^{29,33}$ but it has not been observed or reported in other large case series. ${ }^{27,31,32,34}$ Cerebellar findings are rarely clinically relevant and are not found in isolation; on exam, frank limb ataxia is infrequent. Cerebellar signs, other than extremity dysmetria, such as overshoot dysmetria of the eyes and limbs, or ataxic dysarthria can be found. ${ }^{31}$

\section{Cognition}

Cognitive impairment in Wilson's disease can be subtle, masked by affective involvement and recognized only in retrospect by the family or patient. When present, cognitive impairment falls into two main, not mutually exclusive, categories, a frontal lobe syndrome, or a subcortical dementia. ${ }^{41,42}$ The frontal syndrome may manifest as impulsivity, promiscuity, impaired social judgment, apathy, decreased attention, executive dysfunction with poor planning and decision making, and emotional lability, at an extreme having pseudobulbar features. The subcortical dementia is characterized by slowness of thinking, memory loss, and executive dysfunction, without cortical signs of aphasia, apraxia, or agnosia. ${ }^{41,42}$ Others have suggested that cognitive involvement in Wilson's disease may be primarily related to psychiatric and motor manifestations. ${ }^{43,44}$

\section{Other neurologic features}

Seizures are not an uncommon feature, occurring in approximately $6 \%$ (exceeding the general population frequency by 10 -fold), are rarely the presenting feature, and have been associated with initiation of chelating therapy. ${ }^{45}$ Hyperreflexia, unusual stereotyped movements, and tics have also been reported. ${ }^{29}$

\section{Ophthalmologic manifestations}

Copper deposits in the limbic region of the cornea known a Kayser-Fleischer (KF) rings are seen in nearly $100 \%$ of those with neurologic Wilson's disease, Figure 1. ${ }^{23,33,46}$ In hepatic and presymptomatic Wilson's disease KF rings are present in about $50 \%{ }^{23}$ KF rings are often evident by unaided visual inspection, but slit-lamp evaluation should be performed to verify their presence. KF rings are typically brown to brownish-green in color and are usually more prominent in the superior and inferior regions of the cornea. KF rings are not exclusive to Wilson's disease and can uncommonly be found in other obstructive liver disease. ${ }^{47}$ Sunflower cataracts are seen less commonly than KF rings, present in about $17 \%{ }^{46}$ Sunflower cataracts do not impair vision, cannot be seen with the unaided eye or with an ophthalmoscope, and require slit-lamp evaluation for detection. With treatment, KF rings and sunflower cataracts become less prominent and can disappear. ${ }^{46}$ Other less common ophthalmologic findings include slowing of saccades, impaired upgaze, and strabismus. The absence of nystagmus in Wilson's disease can also be diagnostically useful. ${ }^{46}$

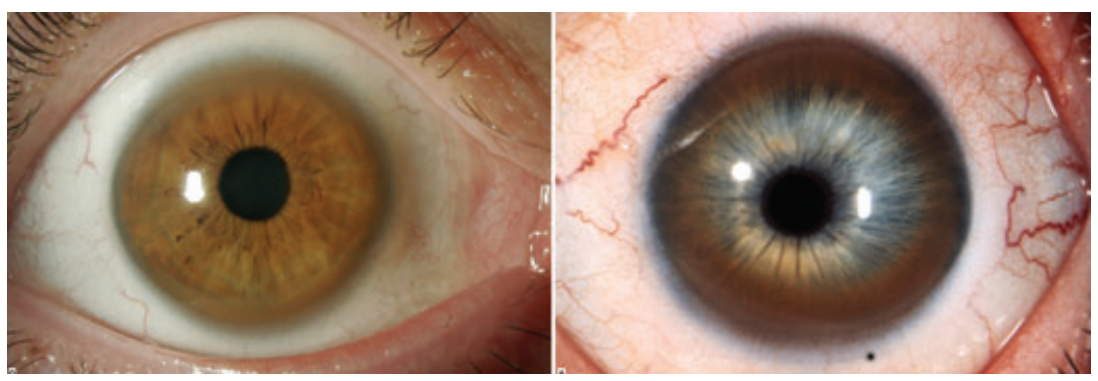

Figure 1. KF rings with prominence at the upper and lower corneal pole in an individual with brown eyes (left panel). Prominent full-circle KF rings in an individual with blue eyes (right panel). 


\section{Initial neurologic features}

Knowledge of the ways neurologic Wilson's disease first manifests is critical to early diagnosis and prompt initiation of treatment. Unfortunately, Wilson's disease is often misdiagnosed, and the average time from symptom onset to diagnostic treatment is long, about 12 months. ${ }^{27,29,31}$ Initial misdiagnosis in Wilson's disease includes over 100 different entities. ${ }^{48}$ Highlighting the diagnostic challenge, initial neurologic misdiagnosis was found to span a spectrum from "no diagnosis," to "neurological problem," and included many common and rare entities. ${ }^{48}$

Diagnostic delay appears clinically significant, as treatment outcomes have been demonstrated to be better in those with a short diagnostic delay. ${ }^{16}$ In patients who made a "very good" recovery, becoming free of neurologic symptoms, 21/57 (37\%) had initiated therapy in less than 1 month. When delay in diagnosis increased to 1-6 months only 7/36 (19\%) were able to achieve a "good" outcome, defined as minor disability. ${ }^{16}$ It has also been shown that a delay in diagnosis increases the likelihood of a poor response to treatment. Walshe and Yealland demonstrated an increasing likelihood of a "poor" response to treatment as delay in diagnosis increased. Poor response was defined as being left with major disability. In those diagnosed in less than 1 month $12 \%$ had a poor outcome, diagnosis in 1-6 months lead to $21 \%$ with poor recovery, a diagnostic delay of
7-18 months led to $19 \%$ with poor outcome, while a greater than 18 -month delay resulted in $38 \%$ with major disability. ${ }^{16}$

The true initial neurologic manifestations of Wilson's disease are illusive, but symptoms and signs at diagnosis have been reported in a number of large case series (Table 1). ${ }^{27,29-31,34}$ In studies that categorize initial manifestations, dysarthria is the most common $(57.6 \%)$, followed by dystonia $(42.4 \%)$, abnormal gait $(37.8 \%)$, tremor $(36.2 \%)$, parkinsonism $(17.3 \%)$, chorea or athetosis $(15.3 \%)$, and seizures $(4.7 \%)$. Ataxia was described as a separate category in only one study. ${ }^{29}$ In these studies, investigations did not use the same criteria to define signs, studies did not use the same categorization, and the proportion of the time symptoms were present in isolation was not specified. Dysarthria, dystonia, tremor, and parkinsonism can be sole initial disease manifestations.

\section{Psychiatric features}

Psychiatric manifestations may be present as often as $30-50 \%$ of the time prior to a diagnosis of Wilson's disease. ${ }^{43,44}$ Because psychiatric symptoms are often ill defined and attributed to other causes, diagnosis of Wilson's disease is rarely made during the period in which psychiatric symptoms are the sole manifestation. This aspect of Wilson's disease is likely a factor in diagnostic and treatment delay. At diagnosis, the most common psychiatric symptoms

Table 1. Initial clinical features of neurologic Wilson's disease

\begin{tabular}{|c|c|c|c|c|c|c|}
\hline & $35^{a}(\%)$ & $30^{b}$ & $28^{c}$ & $31^{d}$ & $32^{c}$ & $\begin{array}{c}\text { Total or } \\
\text { (\%Average) }\end{array}$ \\
\hline Number of patients & 48 & 119 & 136 & 27 & 31 & 361 \\
\hline Age at onset (years) & & 19.6 & 16.2 & 20.9 & 21 & \\
\hline Diagnostic delay (months) & & $\sim 12$ & 12.8 & & $\sim 12$ & \\
\hline Dysarthria & 46 & 91 & 39 & 15 & 97 & $(57.6)$ \\
\hline Dystonia & 38 & 69 & 29 & 11 & 65 & $(42.4)$ \\
\hline Abnormal gait & 24 & 75 & 10 & & 42 & $(37.8)$ \\
\hline Tremor & 22 & 55 & 35 & 37 & 32 & $(36.2)$ \\
\hline parknisonism & 12 & $58^{e}$ & 21 & & 19 & $(17.3)$ \\
\hline Chorea or athetosis & 6 & 30 & & & 10 & $(15.3)$ \\
\hline seizures & 6 & 4.2 & & 4 & & $(4.7)$ \\
\hline Ataxia/cerebellar & & 28 & & & & \\
\hline
\end{tabular}

${ }^{a}$ first manifestations, ${ }^{b}$ at time of diagnosis, ${ }^{c}$ initial signs of neurologic presentation, ${ }^{d}$ initial symptoms of neurologic presentation, ${ }^{e}$ bradykinesia. 
have been reported to be personality change, incongruous behavior, irritability, and depression. ${ }^{44}$ During the course of disease, in addition to persistence of the presenting psychiatric symptoms, other features that can develop include impulsivity, dysinhibition, irritability, reckless behavior, anxiety, substance abuse, catatonia, emotionality, and mania. ${ }^{43,44,49}$ Depression has been estimated to occur in $20-30 \%$ of those affected by Wilson's disease. It has been suggested that depression in Wilson's disease may be reactive, but this does not account for those presenting with depression. Psychotic features do not appear to be a common manifestation of Wilson's disease. ${ }^{44,49}$ Psychiatric manifestations of Wilson's disease appear to be more common with neurologic involvement and are uncommon in the hepatic presentation. ${ }^{44,50}$

\section{Hepatic features of Wilson's disease}

The hepatic presentation can be divided into four main presentations, acute hepatitis, chronic active hepatitis, cirrhosis, and acute fulminant hepatic failure. ${ }^{51}$ Other hepatic presentations include clinical pictures indistinguishable from alcoholic liver disease and autoimmune hepatitis. ${ }^{26}$ A younger onset is seen in those whose symptoms are first hepatic: 11-15 years of age. ${ }^{22,24}$ Because separating hepatitis, cirrhosis, or liver failure due to Wilson's disease from other etiologies is clinically challenging, screening for Wilson's disease should be performed in the appropriate clinical setting. Patients who present with hepatic failure may have mild failure with jaundice, low blood albumin, and edema, but not be in an acute, rapidly deteriorating, fulminant liver failure. Other patients may present with acute fulminant hepatic failure with Coombsnegative hemolytic anemia, coagulopathy, renal failure, and encephalopathy. ${ }^{52}$ Hemolysis is believed to occur secondary to large copper release by acute hepatic necrosis. The occurrence of hemolysis in hepatic disease should always trigger a search for Wilson's disease because it is by far the most likely diagnosis. Patients with a neurologic presentation frequently have subclinical liver disease with a mildto-moderate cirrhosis. For those with further interest, excellent reviews regarding hepatic features of Wilson's disease are available. ${ }^{24,26,53}$

In addition to the neurologic, psychiatric, and hepatic features, patients with Wilson's disease may have a variety of other clinical manifestations. These include abnormalities of renal tubular function, including Fanconi syndrome. Renal stones and gallstones are not uncommon. Patients may have osteoporosis or osteomalacia, or they may have joint disorders such as arthritis or arthralgias. Female patients frequently have oligomenorrhea or amenorrhea. Abnormalities of the heart include interstitial fibrosis and myocarditis. Electrocardiographic abnormalities and orthostatic hypertension are not uncommon. Pancreatic disease, parathyroidism, and skin abnormalities may be present. ${ }^{23}$

\section{Natural history}

Neurologic Wilson's disease most frequently becomes symptomatic in late childhood through early adulthood with a mean age of onset between 15 and 21 years of age..$^{27,29-33}$ It is unusual for Wilson's disease to become symptomatic after age 55 , but a small number have neurologic onset over age 55 (Ref. 54, personal observation). Like the variable combinations of disease manifestations, the clinical course of Wilson's disease is highly variable. Although symptoms are typically gradual in their progression, sudden worsening, both with and without treatment, occurs. Day-to-day fluctuations of symptom severity is observed. It has been suggested that those with tremor-predominant symptoms may have a slower course than those with the predominantly dystonic form. ${ }^{37}$ Younger-onset patents are more likely to have chorea and dystonia, and less likely to have a tremor predominant manifestation, whereas as those with an older onset tend to have tremor as the predominant sign. ${ }^{27}$ Prior to the treatment era, the medial survival following development of neurologic symptoms was approximately $2-5$ years, but Wilson's disease can progress slowly over more than 25 years. ${ }^{16,55}$ In the absence of treatment, neurologic symptoms were progressive and resulted in a severely dystonic akinetic mute state with relative preservation of cognition; untreated Wilson's disease was inevitably fatal. ${ }^{23}$ Experience and detailed investigations convincingly demonstrate the benefit of treatment, with chelation therapy normalizing life expectancy. ${ }^{16-20,24,33}$

\section{Neuropathology}

Wilson described softening of the lenticular nuclei, but it is now realized that brain pathology can 
be widespread and include the thalamus, subthalamic nuclei, brainstem, and frontal cortex. Grossly, the brain often demonstrates atrophy and increased ventricular size. The putamen and caudate can be brown and shrunken. Cavitation and cyst formation in the putamen, and frontal lobes is seen in advanced disease. Spongy degeneration of the cereberal cortex and subcortical white matter, particularly in the frontal lobes, occurs. Microscopic evaluation of affected areas demonstrates neuronal loss, pigment- and lipid-containing macrophages, and gliosis; Opalaski cells in the globus pallidus are a distinctive feature. ${ }^{22,56}$

\section{Molecular genetics and pathogenesis}

The Wilson's disease gene was mapped to chromosome 13q14.3, and the causative gene identified as ATP7B. ${ }^{7-9}$ ATP7B is a 1411 -amino acid, coppertransporting, P-type transmembrane ATPase that is highly expressed in the liver, kidney, and placenta. ${ }^{7}$ Humans, including Wilson's disease patients, take in about $1.0 \mathrm{mg}$ of copper per day in their diet and have a requirement for only about $0.75 \mathrm{mg}$. Thus, the extra $0.25 \mathrm{mg}$ must be eliminated. The normal mechanism for elimination of excess copper is excretion in the bile for loss in the stool. Dietary copper is absorbed in the stomach and duodenum and transported via the portal vein to the liver, the main organ controlling copper regulation. Copper is absorbed into hepatocytes by copper transporter 1, then ATOX1, a copper-specific chaperone protein, transports copper to ATP7B. The normal function of ATP7B appears to be incorporation of copper into ceruloplasmin and secretion of copper into bile. As a result of the mutation in the ATP7B gene, the liver is not capable of excreting excess copper into the bile, and a positive copper balance, averaging about $0.25 \mathrm{mg} / \mathrm{d}$, is established. Copper accumulates over time, first in the liver and then in other parts of the body, such as in the brain. The damage from excessive copper appears to be oxidant in nature. ${ }^{23,53}$

Wilson's disease is an autosomal recessive disease that occurs when a patient carries mutations in both copies of his/her ATP7B gene. Mutational analysis has identified over 300 different mutations throughout the ATP7B gene. A much smaller number of mutations is seen in the majority of patients, and individual mutations have been associated with different ethnic populations. The H1069Q substitution is the most common mutation in white populations representing approximately $40-60 \%$ of identified mutations. ${ }^{57}$ The H1069Q is not prominent in Chinese or Indian populations, in whom other mutations, including R778G, occur at higher frequency. ${ }^{58,59}$ The H1069Q mutation, particularly when homozygous, has been associated with a later onset neurologic disease. ${ }^{60,61}$ Other consistent genotype-phenotype correlations have been elusive. Because of the large number of disease-causing mutations, most patients are compound heterozygotes (have two different mutations). It is not know how the same mutation is able to cause hepatic disease in some, but neurologic in others. A combination of environmental, genetic, and epigenetic factors is likely responsible.

\section{Differential diagnosis}

Neurologic Wilson's disease must be distinguished from other common and rare neurologic diseases. Because Wilson's disease is treatable, it should be considered as a possible diagnosis in every young person with a movement disorder. The more common neurologic disorders that can mimic Wilson's disease include essential tremor, young-onset Parkinson's disease, and generalized dystonia. Rare juvenile genetic extrapyramidal disorders including Huntington disease, Hallervorden-Spatz disease, idiopathic torsion dystonia, chorea-acanthocytosis, and benign familial chorea can at times mimic Wilson's disease. Neither chorea nor ataxia is typically found in isolation, and although Wilson's disease can be considered, diagnostic consideration for other causes of primarily choreic and ataxic diseases should be undertaken. The psychiatric abnormalities may be mistaken for a psychological abnormality, affective disorder, early schizophrenia, or drug abuse. The presence of Kayser-Fleischer rings and the coexistence of liver disease provide important clues.

\section{Diagnostic workup}

The most common screening method for Wilson's disease is a blood ceruloplasmin determination, although this is inadequate for either ruling in or ruling out Wilson's disease. The ceruloplasmin value is usually low in Wilson's disease, but in approximately $10 \%$ of patients it may be normal or near normal. About $10 \%$ of heterozygous carriers who will never have clinical problems will also 
demonstrate low ceruloplasmin values. The most useful screening procedure is a 24 -h urine copper test. ${ }^{22,23}$ In symptomatic Wilson's disease 24 -h urine copper is always elevated to a value greater than $100 \mu \mathrm{g}$ per $24 \mathrm{~h}$ (normal is 50 or less). The $24-\mathrm{h}$ urine sample must be collected in a container free of trace elements. A laboratory capable of measuring copper in low concentrations is required to do the assay. If these difficulties can be overcome, this test is quite reliable in screening for Wilson's disease.

Another common screening procedure is a slitlamp examination for KF rings. Visual inspection is not adequate. KF rings are invariably present in the psychiatric and neurologic presentations; however, they are present in only about $50 \%$ of patients who present with liver disease. In a patient with classical clinical disease, KF rings, and elevated urine copper, the diagnosis can be made with certainty. If any question remains, the gold standard for diagnosis is a measure of quantitative copper in a percutaneous liver biopsy. The hepatic copper value in untreated Wilson's disease is above $200 \mu \mathrm{g} / \mathrm{g}$ dry weight of tissue, with normal being $50 \mu \mathrm{g} / \mathrm{g}$ or less. Carriers of the Wilson's disease gene may have mild elevations of hepatic copper, but never to $200 \mu \mathrm{g} / \mathrm{g}$. It is important not to rely on the stain for copper, for if the copper is still diffusely cytoplasmic, the copper stain may be negative in the face of very great elevations of hepatic copper. Radiocopper studies have been suggested for the diagnosis of Wilson's disease, but their usefulness is questionable. While these tests are abnormal in Wilson's disease, they are also abnormal in carriers of the disease and thus are fraught with the risk of misdiagnosis. ${ }^{23}$

The gene for Wilson's disease has been cloned, ${ }^{7-9}$ fostering hope for the development of a direct DNA test. This approach is not practical because more than 300 different mutations that may cause Wilson's disease have been identified. ${ }^{53,57}$ However, if a mutation is identified in a proband, then search for this mutation in relatives can provide crucial information for the identification of as yet asymptomatic relatives and of heterozygotes, and provides the opportunity for preventive therapy and genetic counseling.

Central nervous system imaging can be helpful in the diagnosis, Figure 2. MRI scans generally demonstrate abnormalities in patients with neurologic or psychiatric symptoms but are often normal in patients with only liver disease. In neurologically in- volved patients, the most common findings are areas of high T2 signal in the lentiform and caudate nuclei, thalamus, brain stem, and white matter. ${ }^{31,62,63}$ High signal T1 images, like those in portal-systemic encephalopathy may also be observed. ${ }^{64}$ Dopamine D2 receptor binding and regional cerebral glucose metabolism are reduced in the striatum and have been shown to return to almost normal levels after penicillamine therapy. ${ }^{65}$

\section{Treatment}

Wilson's disease is a condition that can be effectively treated. Treatment can be divided into initial therapy, maintenance therapy, and treatment of the presymptomatic patient. ${ }^{23,26}$ Available pharmacologic agents include penicillamine, trientine, zinc acetate, and tetrathiomolybdate. The aim of treatment is to reduce the amount of toxic free copper. Free copper is toxic whereas copper bound to ceruloplasmin or metallothionein is not.

Although penicillamine demonstrated clear therapeutic advantages over BAL, initial neurologic worsening following initiation of penicillamine therapy is a concern. In their retrospective study, Brewer and colleagues found that the risk for neurologic worsening was about $50 \%$ when penicillamine was used as initial treatment of neurologic Wilson's disease and that $50 \%$ of those who deteriorated never recovered to their prepenicillamine baseline. ${ }^{66}$ The majority of patients observed to have deteriorated did so within 4 weeks of beginning penicillamine therapy. It has been suggested that mobilization of large hepatic copper stores raises blood free-copper levels leading to increased toxic copper exposure to the brain and subsequent worsening. ${ }^{66}$

Others have investigated the efficacy and toxicity of penicillamine treatment of neurologic Wilson's disease. ${ }^{24,32,67}$ In a description of their experience Walshe and colleagues described outcomes following treatment of neurologic Wilson's disease with penicillamine. In this study if toxicity developed while using penicillamine, therapy was switched to trientine. In some patients in this series, trientine was used as first-line therapy, and in a small number zinc, BAL, or tetrathiomolybdate was used following penicillamine. ${ }^{16}$ In all, 137 patients were followed, 57 (41\%) became symptom free, $36(26 \%)$ were left with minor neurologic deficit, $24(18 \%)$ were left disabled, and 20 


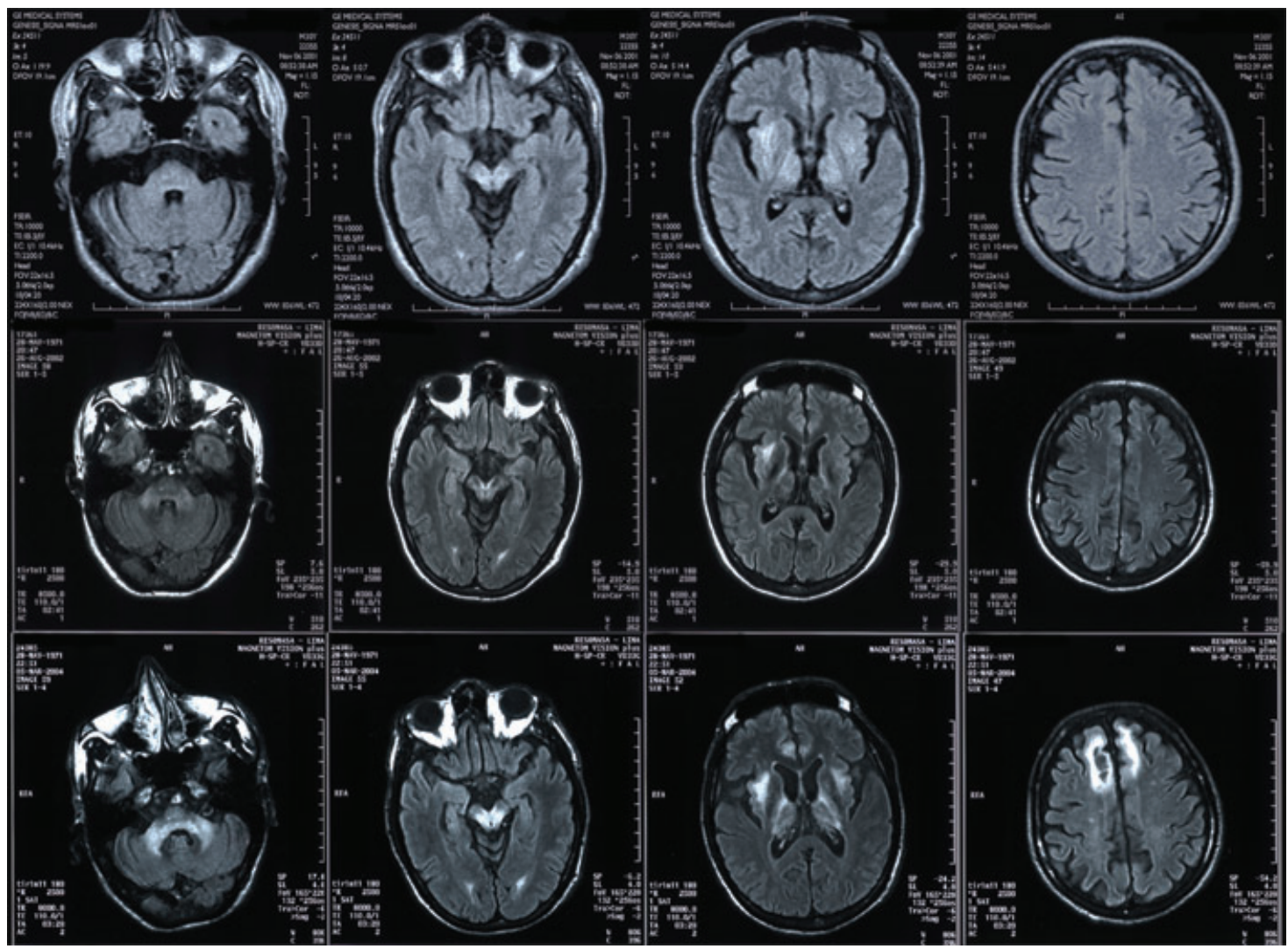

Figure 2. Progressive MRI changes as shown in sequential brain fluid-attenuated inversion-recovery (FLAIR) imaging in an individual with untreated Wilson's disease. Representative images are shown at the level of the superior cerebellar peduncle ( first column), the midbrain (second column from the left), the basal ganglion (third column from the left), and at the level of the cerebral cortex (right-most column). Images in the top, middle, and bottom rows were obtained in 2001, 2002, and 2004, respectively.

(15\%) died. ${ }^{16}$ Most patients who improved began to do so in 3-6 months. Thirty (22\%) had increased symptom severity after initiation of treatment. In those who had initial worsening, 11 (37\% of those who worsened) eventually became symptom free, $9(30 \%)$ were left with minor neurologic deficit, and $10(33 \%)$ were left disabled. ${ }^{16}$ No features were identified that were able to predict those who worsened. Of individuals who died despite treatment, no factors could be identified to predict who would die. These patients were not more likely to present with a dystonic phenotype, but most had became severely dystonic prior to death. ${ }^{16}$ In this study penicillamine was associated with toxicity requiring discontinuation of its use in around $20 \% .{ }^{16}$ In another study of largely neurologic Wilson's disease $44 \%$ discontinued penicillamine secondary to side effects. ${ }^{68}$ Complications of penicillamine include rash, immune complex nephropathy, systemic lupus erythematosus, thrombocytopenia, and leucocytopenia. ${ }^{16,68}$

This study is additionally notable because neurologic features were examined for an association with treatment outcome. The pseudosclerotic (tremor and dysarthric) form of neurologic Wilson's disease was found to be associated with the best chance of a favorable outcome. ${ }^{16}$ Patients with the pseudosclerotic form of neurologic Wilson's disease were found to have an $82 \%$ chance of becoming symptom free or having minor neurologic deficit. Those with chorea had a $75 \%$ chance, those with parkinsonism a $63 \%$ chance, and those with dystonia a $53 \%$ chance of becoming symptom free or having minor neurologic deficit. $^{16}$ 
Stremmel and colleagues described their experience with penicillamine in a total of 51 patients, 31 with neurologic Wilson's disease. ${ }^{32}$ In this study neurologic and hepatic Wilson's were often considered together. Improvement in neurologic features was described following penicillamine therapy. No description of initial neurologic worsening was reported, but penicillamine-induced adverse reactions occurred in $25 \% .^{32}$ Stremmel and colleagues further described treatment of Wilson's disease in 163 additional Wilson's disease patients, 58 with neurologic Wilson's disease. ${ }^{24}$ In the neurologic group, $54 \%$ improved, $22 \%$ were stable, and $24 \%$ worsened. Of all the patients $70.3 \%$ developed side effects to penicillamine and $31 \%$ experienced severe side effects including neurologic worsening. Neurologic worsening occurred early after initiation of therapy and in some did not improve. ${ }^{24}$

Because of concern for neurologic worsening in those treated with penicillamine, use of other agents has been recommended for initial treatment of neurologic Wilson's disease. ${ }^{14,20,24,69}$ If penicillamine is used, the standard starting dose is $250 \mathrm{mg}$ four times a day or $500 \mathrm{mg}$ twice a day. It has been suggested that starting with lower doses, 250-500 mg per day for a few weeks may lessen side effects. ${ }^{26}$ When initiated, the patient should be carefully monitored for toxicities including to the bone marrow and kidney. An acute hypersensitivity reaction occurs in about $25 \%$ of patients, which may respond to corticosteroid therapy or withdrawal of the drug and readministration in very low doses.

Trientine has often been used as an alternative or second-line therapy, but like penicillamine it can cause neurologic worsening in approximately $25 \% .^{20,26}$ Trientine is a chelator that, like penicillamine, promotes urinary excretion of copper. Trientine is used in doses of 750-1500 mg for initial therapy and $750 \mathrm{mg}$ to $1000 \mathrm{mg}$ for maintenance therapy, divided into two or three doses per day. It should be given $1 \mathrm{~h}$ before or $2 \mathrm{~h}$ after meals. Although trientine has fewer side effects than penicillamine it shares a common mechanism of action and can cause neurologic worsening. In a double-blind study comparing tetrathiomolybdate to trientine, $24 \%$ of those treated with trientine experienced neurologic worsening and in those who initially worsened $50 \%$ died. ${ }^{20}$ While on trientine, urine copper is a reflection of enhanced urinary copper excretion and total body copper load. Upon initial treatment with trientine, 24-h urinary copper excretion may be $1000-3000 \mu \mathrm{g}$ (normal is $20-50 \mu \mathrm{g} / 24 \mathrm{~h}$ ). After a few weeks urinary copper decreases to 500 $1000 \mu \mathrm{g}$ per $24 \mathrm{~h}$, and after approximately 1 year of therapy it should decrease to $200-500 \mu \mathrm{g}$ per $24 \mathrm{~h}$. Nonceruloplasmin-bound copper, free copper, is a useful measure for monitoring efficacy of trientine and can be calculated by the formula: (Total serum copper in $\mu \mathrm{g} / \mathrm{mL} \times 100$ ) - (ceruloplasmin in $\mathrm{mg} / \mathrm{dL}$ $\times 3$ ) $=$ free copper (normal range is $5-15 \mu \mathrm{g} / \mathrm{dL}$ ). Another way that free copper can be accurately estimated is by subtracting $3 \mu \mathrm{g}$ for every $1 \mathrm{mg} / \mathrm{dL}$ of ceruloplasmin from the serum copper, expressed as $\mu \mathrm{g} / \mathrm{dL}$. During the maintenance phase of trientine therapy keeping free copper below $25 \mathrm{mg}$ is the goal.

Zinc acetate therapy has been used successfully as a preventative therapy in presymptomatic patients, as initial treatment for neurologic Wilson's disease, and as maintenance therapy following an initial course of decoppering. ${ }^{69,70}$ Zinc induces intestinal cell metallothionein, a protein that complexes intestinal food copper or endogenously secreted copper in saliva and gastric secretions. The complex cannot be absorbed in to the blood, and as intestinal cells die they, along with the complex, are sloughed into the stool resulting in a negative copper balance. The net effect of zinc therapy is an intestinal blockade of zinc absorption. Zinc has the additional effect of inducing hepatocyte metallothionein production, which may reduce the toxic effect of free copper in these cells. ${ }^{22,70}$

The decoppering effect of zinc is slow: a period of 4-8 months of treatment is required to reduce copper to nontoxic levels. In symptomatic individuals, the natural course of disease continues during this period and can lead to permanent worsening. To avoid potential toxicities of trientine and penicillamine, some have successfully used zinc in the initial treatment of neurologic Wilson's disease. ${ }^{15,69,71-75}$ An advantage of zinc is its lack of serious significant side effects and safety in long-term use. ${ }^{71,76}$ Approximately $10 \%$ experience gastric discomfort or nausea upon initiation of zinc therapy. The use of the zinc acetate compared to zinc sulfate reduces gastric discomfort. Generally, gastric symptoms subside within days to weeks. For maintenance and prophylactic therapy in adults, $50 \mathrm{mg}$ of zinc three times a day is recommended. ${ }^{69,70,76}$ It is important to separate the zinc from food by at least an hour. For maintenance therapy zinc is 
favored over penicillamine or trientine, because while all three drugs are effective, zinc has a superior side-effect profile and does not appear to cause neurologic worsening. ${ }^{23,69}$ Because zinc therapy does not induce urinary excretion of copper, as do trientine or penicillamine, urine copper is an accurate reflection of body copper stores. Following a year of zinc therapy, a 24-h copper of around $125 \mu \mathrm{g}$ is a reflection of good copper control. Compliance with zinc can be assessed by monitoring 24 -h urine zinc excretion, which should be above $2 \mathrm{mg} .{ }^{22}$

For the initial treatment of patients presenting with neurologic or psychiatric disease, the use of tetrathiomolybdate (TM) followed by zinc maintenance therapy has been suggested to provide a good best balance of efficacy and side effects, but TM is not yet FDA approved. ${ }^{20} \mathrm{TM}$ acts by forming a tripartite complex with copper and protein. Given with food, TM binds food copper and endogenously secreted copper preventing copper absorption. Given between meals, TM is absorbed into the blood and forms a complex with free copper and albumin. Free copper in the blood is in equilibrium with tissue copper so that binding of serum copper to TM shifts the equilibrium to mobilize copper from tissue stores into serum where it is bound by TM and excreted into bile. The result is a rapid removal of tissue copper, which is then bound in the serum by TM, reducing potential copper toxicity, and significantly less neurologic worsening compared to penicillamine or trientine. ${ }^{17-20}$ In a double-blind study 48 patients were randomized to TM or trientine for the initial treatment of neurologic Wilson's disease. TM, $20 \mathrm{mg}$ three times a day with meals and $20 \mathrm{mg}$ three times a day between meals, or trientine, $500 \mathrm{mg}$ two times per day, were given for 8 weeks with zinc, $50 \mathrm{mg}$ twice daily, followed by zinc maintenance therapy. Both trientine and TM were found to improve neurologic and speech function, but TM was significantly less likely to be associated with neurologic worsening than trientine. ${ }^{20}$ In the trientine arm 6 of $23(26 \%)$ patients worsened neurologically, and 3 of those who worsened died. In the TM arm 1 of $25(4 \%)$ patients developed neurologic deterioration, a statistically significant difference. In an earlier open-label study of TM in initial treatment of neurologic Wilson's disease 2 of 55 (3.6\%) patients treated with TM worsened. ${ }^{19}$ Serious adverse effects from TM were not observed. TM was associated with reversible increases in transaminase levels and anemia in about $15 \% .{ }^{19,20}$ Anemia and transaminase elevations responded to temporary suspension of TM without subsequent abnormalities following reinitiation of TM at half dose. ${ }^{19,20} 24$-h urine copper monitoring during treatment with TM can be used to monitor therapy. In untreated Wilson's disease, 24-h urinary excretion always exceeds $100 \mu \mathrm{g}$ per $24 \mathrm{~h}$. The goal of treatment following an initial course of TM followed by maintenance therapy with zinc is a $24 \mathrm{~h}$ urine copper excretion of $50-100 \mu \mathrm{g}$. A study evaluating lower doses of TM is ongoing (G.J. Brewer, personal communication, 2008). It is anticipated that TM will be FDA approved, and more widely available.

Some have reported that individuals with decompensated liver disease treated with orthotopic liver transplant demonstrated improvement of neurologic symptoms. ${ }^{77,78}$ Other studies have not demonstrated a benefit, and orthotopic liver transplant is not recommended as a treatment for neurologic Wilson's disease. ${ }^{22,79}$ In those studies reporting on the effect of treatment, the largest degree of improvement was observed between 6 months and 2 years of adequate copper control, with some benefit reported to occur for up to 3 years. ${ }^{20,24,32,43,44,67}$ The response to treatment is variable. Few studies have systematically investigated or reported the effect of treatment on individual neurologic signs or symptoms. Currently no reliable predictors exist to differentiate those who will make an excellent recovery from those whose symptoms will stabilize but not improve. It has been suggested that tremor may be more treatment responsive than dystonia and dysarthria. ${ }^{16,36}$ Those who did not respond to treatment tended to have a more severe neurologic disease, with more frequent dysarthria and dystonia. ${ }^{16,44}$ In those severely impaired by neurologic Wilson's disease, attempts to determine factors associated with a good recovery were not found. ${ }^{67}$

Symptomatic treatment of the movement disorders found in Wilson's patients has not be carefully studied, and response to treatment is inconsistent. ${ }^{16}$ There are no good studies that have investigated dopamine agonists for treatment of parkinsonism in Wilson's disease. L-dopa and dopamine agonists do not appear to provide reliable relief of parkinsonism. $\beta$-blockers and primidone, medications used to treat essential tremor, can be tried in those with limiting essential tremor-like tremor, but their benefit is limited. Artane, baclofen, and valium can be used in 
symptomatic therapy of dystonia. Speech and physical therapy can provide a great deal of benefit.

Psychiatric manifestations of Wilson's disease also have a variable response to treatment. Incongruous behavior and cognitive impairment were reported to be more treatment responsive than irritability or depression. Improvements tended to occur relatively early in the course of treatment, before 3.5 years, and then reach a plateau. ${ }^{44}$ Others have suggested that the most prominent improvement occurs between 6 and 18 months following initiation of therapy and then plateaus after 2 years of copper-level normalization. ${ }^{43}$

In patients who present with liver disease, if the disease if fulminant, hepatic transplant may be the only way to save the patient's life. However, in cases of mild hepatic failure, a picture of chronic cirrhosis, or a picture of mild hepatitis, medical treatment can be quite effective. The prognostic index of Nazer is useful in differentiating which patients will likely do well with medical therapy. ${ }^{80}$ The combination of trientine followed by zinc maintenance may represent the best medical treatment for hepatic Wilson's disease. The trientine is given to obtain a rather robust negative copper balance. Zinc is given to induce hepatic metallothionein and allow that metallothionein to complex copper in the liver in a nontoxic form. This combination is used for 4 months and then the trientine is discontinued.

Over the years, considerable attention has been paid to diet in Wilson's disease. In the United States only liver and shellfish are high enough in copper to be of concern. ${ }^{22,23}$ Because animals are fed high levels of minerals, the liver can be very high in copper and should not be ingested during the decoppering period (first 4 months) and after that only in small amounts. Shellfish are intermediately high in copper and should not be ingested during the decoppering period and not ingested more often than once a week during maintenance therapy. Other foods are not restricted because the content of copper is not high enough to be of concern. Occasionally, drinkingwater samples will be found to be high in copper. If the level is higher than about $0.1 \mathrm{ppm}$ the patient should use an alternative source of drinking water.

\section{Prevention}

Identification and treatment of presymptomatic Wilson's disease patients can prevent development of symptoms. Aggressive screening measures in clinic populations at risk are critical. An important target population is the siblings of the newly diagnosed patient. Each sibling has a $25 \%$ risk of being in the presymptomatic disease stage. Because prophylactic therapy will prevent the onset of the disease, these patients should be examined and disease status determined. All full siblings should be screened for blood ceruloplasmin, and 24-h urine copper levels. A 24-h urine copper in presymptomatic siblings with a value of 100 is diagnostic of Wilson's disease. If it is less than 50 in an adult patient, it essentially excludes the diagnosis. If it is intermediate, it is compatible either with the carrier state, since some of these patients have mild elevations of urine copper, or the presymptomatic affected state, since some of these patients do not have diagnostic levels of urine copper. The patients in this intermediate zone should have a liver biopsy to make the diagnosis. The liver copper level in such a patient is diagnostic. Because the risk of Wilson's disease is significantly elevated in nieces and nephews (1/600) and cousins (1/800), compared to the general population, these relatives can be screened for ceruloplasmin and $24-h$ urine copper levels.

\section{Conclusion}

The history of Wilson's disease is remarkable in many aspects. Like other well known neurodegenerative diseases such as Alzheimer's or Parkinson's disease, pioneering individuals contributed seminal observations regarding disease features and etiology. Unlike other neurodegenerative diseases this work has lead to treatment that can essentially cure the disease. Unfortunately, neurologic Wilson's disease continues to suffer from clinically significant diagnostic delay. Currently available therapies have had a major positive impact on the outcome of neurologic Wilson's disease, but have limitations. Future studies promise to delineate optimal therapeutic regimes and improved clinical outcomes.

\section{Acknowledgments}

The author would like to thank Dr. Wayne Cornblath for providing the KF ring images. The author would also like to acknowledge Dr. George Brewer for his continued commitment to Wilson's disease patients and for providing me the opportunity to be involved in his research. 


\section{Conflicts of interest}

The author declares no conflicts of interest.

\section{References}

1. Wilson, S. 1912. Progressive lenticular degeneration: A familial nervous disease associated with cirrhosis of the liver. Brain 34: 295-509.

2. Walshe, J.M. 1988. Wilson's disease: yesterday, today, and tomorrow. Mov. Disord. 3: 10-29.

3. Scheinberg, I.H. \& D. Gitlin. 1952. Deficiency of ceruloplasmin in patients with hepatolenticular degeneration (Wilson's disease). Science 116: 484-485.

4. Osborn, S.B., C.N. Roberts \& J.M. Walshe. 1963. Uptake of radiocopper by the liver. A study of patients with Wilson's disease and various control groups. Clin. Sci. 24: 13-22.

5. Walshe, J.M. \& G. Potter. 1977. The pattern of the whole body distribution of radioactive copper $(67 \mathrm{Cu}, 64 \mathrm{Cu})$ in Wilson's Disease and various control groups. Q. J. Med. 46: 445-462.

6. Bearn, A.G. 1960. A genetical analysis of thirty families with Wilson's disease (hepatolenticular degeneration). Ann. Hum. Genet. 24: 33-43.

7. Bull, P.C. et al. 1993. The Wilson disease gene is a putative copper transporting P-type ATPase similar to the Menkes gene. Nat. Genet. 5: 327-337.

8. Tanzi, R.E. et al. 1993. The Wilson disease gene is a copper transporting ATPase with homology to the Menkes disease gene. Nat. Genet. 5: 344-350.

9. Yamaguchi, Y., M.E. Heiny \& J.D. Gitlin. 1993. Isolation and characterization of a human liver cDNA as a candidate gene for Wilson disease. Biochem. Biophys. Res. Commun. 197: 271-277.

10. Cumings, J.N. 1948. The copper and iron content of brain and liver in the normal and in hepato-lenticular degeneration. Brain 71: 410-415.

11. Cumings, J.N. 1951. The effects of B.A.L. in hepatolenticular degeneration. Brain 74: 10-22.

12. Denny-Brown, D. \& H. Porter. 1951. The effect of BAL (2,3-dimercaptopropanol) on hepatolenticular degeneration (Wilson's disease). N. Engl. J. Med. 245: 917-925.

13. Walshe, J.M. 1956. Penicillamine, a new oral therapy for Wilson's disease. Am. J. Med. 21: 487-495.

14. Brewer, G.J. et al. 1983. Oral zinc therapy for Wilson's disease. Ann. Intern. Med. 99: 314-319.

15. Hoogenraad, T.U., R. Koevoet \& E.G. de Ruyter Korver. 1979. Oral zinc sulphate as long-term treatment in Wilson's disease (hepatolenticular degeneration). Eur. Neurol. 18: 205-211.
16. Walshe, J.M. \& M. Yealland. 1993. Chelation treatment of neurological Wilson's disease. Q. J. Med. 86: 197204.

17. Brewer, G.J. et al. 1994. Treatment of Wilson's disease with ammonium tetrathiomolybdate. I. Initial therapy in 17 neurologically affected patients. Arch. Neurol. 51: 545-554.

18. Brewer, G.J. et al. 1996. Treatment of Wilson disease with ammonium tetrathiomolybdate. II. Initial therapy in 33 neurologically affected patients and follow-up with zinc therapy. Arch. Neurol. 53: 1017-1025.

19. Brewer, G.J. et al. 2003. Treatment of Wilson disease with ammonium tetrathiomolybdate: III. Initial therapy in a total of 55 neurologically affected patients and follow-up with zinc therapy. Arch. Neurol. 60: 379-385.

20. Brewer, G.J. et al. 2006. Treatment of Wilson disease with ammonium tetrathiomolybdate: IV. Comparison of tetrathiomolybdate and trientine in a double-blind study of treatment of the neurologic presentation of Wilson disease. Arch. Neurol. 63: 521-527.

21. Reilly, M., L. Daly \& M. Hutchinson. 1993. An epidemiological study of Wilson's disease in the Republic of Ireland. J. Neurol. Neurosurg. Psychiatry 56: 298-300.

22. Brewer, G.J. 2001. Wilson's Disease : A Clinician's Guide to Recognition, Diagnosis, and Management. Kluwer Academic. Boston.

23. Brewer, G.J. \& V. Yuzbasiyan-Gurkan. 1992. Wilson disease. Medicine (Baltimore) 71: 139-164.

24. Merle, U. et al. 2007. Clinical presentation, diagnosis and long-term outcome of Wilson's disease: a cohort study. Gut 56: 115-120.

25. Ala, A. et al. 2005. Wilson disease in septuagenarian siblings: Raising the bar for diagnosis. Hepatology 41: 668-670.

26. Roberts, E.A. \& M.L. Schilsky. 2003. A practice guideline on Wilson disease. Hepatology 37: 1475-1492.

27. Walshe, J.M. \& M. Yealland. 1992. Wilson's disease: the problem of delayed diagnosis. J. Neurol. Neurosurg. Psychiatry 55: 692-696.

28. Giagheddu, A. et al. 1985. Epidemiologic study of hepatolenticular degeneration (Wilson's disease) in Sardinia (1902-1983). Acta Neurol. Scand. 72: 43-55.

29. Machado, A. et al. 2006. Neurological manifestations in Wilson's disease: Report of 119 cases. Mov. Disord. 21: 2192-2196.

30. Oder, W. et al. 1991. Neurological and neuropsychiatric spectrum of Wilson's disease: a prospective study of 45 cases. J. Neurol. 238: 281-287.

31. Starosta-Rubinstein, S. et al. 1987. Clinical assessment of 31 patients with Wilson's disease. Correlations with 
structural changes on magnetic resonance imaging. Arch. Neurol. 44: 365-370.

32. Stremmel, W. et al. 1991. Wilson disease: clinical presentation, treatment, and survival. Ann. Intern. Med. 115: 720-726.

33. Taly, A.B. et al. 2007. Wilson disease: description of 282 patients evaluated over 3 decades. Medicine (Baltimore) 86: 112-121.

34. Soltanzadeh, A. et al. 2007. Wilson's disease: a great masquerader. Eur. Neurol. 57: 80-85.

35. Dening, T.R. \& G.E. Berrios. 1989. Wilson's disease: clinical groups in 400 cases. Acta Neurol. Scand. 80: 527-534.

36. Denny-Brown, D. 1964. Hepatolenticular degeneration (Wilson's disease). Two different components. N. Engl. J. Med. 270: 1149-1156.

37. Okinaka, S. 1961. Studies on hepatocerebral disease. III. Hepatolenticular degeneration in Japan, with studies on copper metabolism. Neurology 11: 792-799.

38. Svetel, M. et al. 2001. Dystonia in Wilson's disease. Mov. Disord. 16: 719-723.

39. Volkmann, J. et al. 1992. Impairment of temporal organization of speech in basal ganglia diseases. Brain Lang. 43: 386-399.

40. Lorincz, M.T. 2006. Geriatric chorea. Clin. Geriatr. Med. 22: 879-897, vii.

41. Lang, C. 1989. Is Wilson's disease a dementing condition? J. Clin. Exp. Neuropsychol. 11: 569-570.

42. Lang, C. et al. 1990. Neuropsychological findings in treated Wilson's disease. Acta Neurol. Scand. 81: 75-81.

43. Akil, M. \& G.J. Brewer. 1995. Psychiatric and behavioral abnormalities in Wilson's disease. Adv. Neurol. 65: 171178.

44. Dening, T.R. \& G.E. Berrios. 1990. Wilson's disease: a longitudinal study of psychiatric symptoms. Biol. Psychiatry 28: 255-265.

45. Dening, T.R., G.E. Berrios \& J.M. Walshe. 1988. Wilson's disease and epilepsy. Brain 111 (Pt 5): 1139-1155.

46. Wiebers, D.O., R.W. Hollenhorst \& N.P. Goldstein. 1977. The ophthalmologic manifestations of Wilson's disease. Mayo Clin. Proc. 52: 409-416.

47. Fleming, C.R. et al. 1977. Pigmented corneal rings in non-Wilsonian liver disease. Ann. Intern. Med. 86: 285288.

48. Prashanth, L.K. et al. 2004. Wilson's disease: diagnostic errors and clinical implications. J. Neurol. Neurosurg. Psychiatry 75: 907-909.

49. Goldstein, N.P. et al. 1968. Psychiatric aspects of Wilson's disease (hepatolenticular degeneration): results of psychometric tests during long-term therapy. Am. J. Psychiatry 124: 1555-1561.
50. Dening, T.R. \& G.E. Berrios. 1989. Wilson's disease. Psychiatric symptoms in 195 cases. Arch. Gen. Psychiatry 46: 1126-1134.

51. Sallie, R. et al. 1992. Failure of simple biochemical indexes to reliably differentiate fulminant Wilson's disease from other causes of fulminant liver failure. Hepatology 16: 1206-1211.

52. Ostapowicz, G. et al. 2002. Results of a prospective study of acute liver failure at 17 tertiary care centers in the United States. Ann. Intern. Med. 137: 947-954.

53. Ala, A. et al. 2007. Wilson's disease. Lancet 369: 397-408.

54. Ross, M.E. et al. 1985. Late-onset Wilson's disease with neurological involvement in the absence of KayserFleischer rings. Ann. Neurol. 17: 411-413.

55. Shimoji, A. et al. 1987. Wilson's disease with extensive degeneration of cerebral white matter and cortex. Jpn. J. Psychiatry Neurol. 41: 709-717.

56. Ellison, D. \& S. Love. 1998. Neuropathology. Mosby. Tokyo.

57. Shah, A.B. et al. 1997. Identification and analysis of mutations in the Wilson disease gene (ATP7B): population frequencies, genotype-phenotype correlation, and functional analyses. Am. J. Hum. Genet. 61: 317328.

58. Gu, Y.H. et al. 2003. Mutation spectrum and polymorphisms in ATP7B identified on direct sequencing of all exons in Chinese Han and Hui ethnic patients with Wilson's disease. Clin. Genet. 64: 479-484.

59. Kumar, S. et al. 2005. Identification and molecular characterization of 18 novel mutations in the ATP7B gene from Indian Wilson disease patients: genotype. Clin. Genet. 67: 443-445.

60. Stapelbroek, J.M. et al. 2004. The H1069Q mutation in ATP7B is associated with late and neurologic presentation in Wilson disease: results of a meta-analysis. $J$. Hepatol. 41: 758-763.

61. Vrabelova, S. et al. 2005. Mutation analysis of the ATP7B gene and genotype/phenotype correlation in $227 \mathrm{pa}-$ tients with Wilson disease. Mol. Genet. Metab. 86: 277285.

62. Magalhaes, A.C. et al. 1994. Wilson's disease: MRI with clinical correlation. Neuroradiology 36: 97-100.

63. van Wassenaer-van Hall, H.N. et al. 1996. Wilson disease: findings at MR imaging and CT of the brain with clinical correlation. Radiology 198: 531-536.

64. Sinha, S.S. et al. 2007. Sequential MRI changes in Wilson's disease with de-coppering therapy: a study of 50 patients. Br. J. Radiol. 80: 744-749.

65. Schlaug, G. et al. 1994. Dopamine D2 receptor binding and cerebral glucose metabolism recover after 
D-penicillamine-therapy in Wilson's disease. J. Neurol. 241: 577-584.

66. Brewer, G.J. et al. 1987. Worsening of neurologic syndrome in patients with Wilson's disease with initial penicillamine therapy. Arch. Neurol. 44: 490-493.

67. Prashanth, L.K. et al. 2005. Prognostic factors in patients presenting with severe neurological forms of Wilson's disease. QJM 98: 557-563.

68. Czlonkowska, A., J. Gajda \& M. Rodo. 1996. Effects of long-term treatment in Wilson's disease with Dpenicillamine and zinc sulphate. J. Neurol. 243: 269273.

69. Hoogenraad, T.U. 2006. Paradigm shift in treatment of Wilson's disease: zinc therapy now treatment of choice. Brain Dev. 28: 141-146.

70. Brewer, G.J., V. Yuzbasiyan-Gurkan \& D.Y. Lee. 1990. Use of zinc-copper metabolic interactions in the treatment of Wilson's disease. J. Am. Coll. Nutr. 9: 487-491.

71. Marcellini, M. et al. 2005. Treatment of Wilson's disease with zinc from the time of diagnosis in pediatric patients: a single-hospital, 10-year follow-up study. J. Lab. Clin. Med. 145: 139-143.

72. Hockly, E. et al. 2002. Environmental enrichment slows disease progression in R6/2 Huntington's disease mice. Ann. Neurol. 51: 235-242.
73. Hoogenraad, T.U. 1998. Zinc treatment of Wilson's disease. J. Lab. Clin. Med. 132: 240-241.

74. Hoogenraad, T.U. \& J. Van Hattum. 1988. Zinc therapy as the initial treatment for Wilson's disease. Arch. Neurol. 45: 373-374.

75. Hoogenraad, T.U., J. Van Hattum \& C.J. Van Den Hamer. 1987. Management of Wilson's disease with zinc sulphate. Experience in a series of 27 patients. J. Neurol. Sci. 77: 137-146.

76. Anderson, L.A., S.L. Hakojarvi \& S.K. Boudreaux. 1998. Zinc acetate treatment in Wilson's disease. Ann. Pharmacother. 32: 78-87.

77. Eghtesad, B. et al. 1999. Liver transplantation for Wilson's disease: a single-center experience. Liver Transpl. Surg. 5: 467-474.

78. Schumacher, G. et al. 1997. Liver transplantation: treatment of choice for hepatic and neurological manifestation of Wilson's disease. Clin. Transplant. 11: 217224.

79. Rodriguez, R.T. et al. 2007. Manipulation of OCT4 levels in human embryonic stem cells results in induction of differential cell types. Exp. Biol. Med. (Maywood) 232: 1368-1380.

80. Nazer, H. et al. 1986. Wilson's disease: clinical presentation and use of prognostic index. Gut 27: 1377-1381. 